\title{
Structure-guided protein engineering of ammonia lyase for efficient synthesis of sterically bulky unnatural amino acids
}

\author{
Zi-Fu Ni, Pei Xu, Min-Hua Zong and Wen-Yong Lou* (i)
}

\begin{abstract}
Enzymatic asymmetric amination addition is seen as a promising approach for synthesizing amine derivatives, especially unnatural amino acids, which are valuable precursors to fine chemicals and drugs. Despite the broad substrate spectrum of methylaspartate lyase (MAL), some bulky substrates, such as caffeic acid, cannot be effectively accepted. Herein, we report a group of variants structurally derived from Escherichia coli O157:H7 MAL (ECMAL). A combined mutagenesis strategy was used to simultaneously redesign the key residues of the entrance tunnel and binding pocket to explore the possibility of accepting bulky substrates with potential application to chiral drug synthesis. Libraries of residues capable of lining the active center of ECMAL were then constructed and screened by an effective activity solid-phase color screening method using tyrosinase as a cascade catalyst system. Activity assays and molecular dynamics studies of the resultant variants showed that the substrate specificity of ECMAL was modified by adjusting the polarity of the binding pocket and the degree of flexibility of the entrance tunnel. Compared to M3, the optimal variant M8 was obtained with a 15-fold increase in catalytic activity. This structure-based protein engineering of ECMAL can be used to open new application directions or to develop practical multi-enzymatic processes for the production of various useful compounds.
\end{abstract}

Keywords: Biocatalysis, Methyl aspartate lyase, Substrate specificity, Protein engineering, Solid-phase color screening

\section{Introduction}

The hydroamination of olefins and carbon-nitrogen bond-forming reactions of unsaturated carboxylic acids offer a vast range of applications in the synthesis of fine chemicals (Raj et al. 2012). However, under classical conditions, the catalysts used are potentially hazardous to the environment. With increasing emphasis on the concept of green, clean, and sustainable chemistry, alternative strategies, such as biological methods, have been proposed for implementing such reactions (Wu et al. 2020). Enzymatic transformation of unsaturated carboxylic acids is an ideal approach for the production

\footnotetext{
${ }^{*}$ Correspondence: wylou@scut.edu.cn

Laboratory of Applied Biocatalysis, School of Food Science

and Engineering, South China University of Technology, No. 381 Wushan

Road, Guangzhou 510640, Guangdong, China
}

of unnatural amino acids, having prominent advantages of economy and sustainability (Zhang et al. 2020a). Six classes of enzymes have been applied for this purpose, as exemplified by $\omega$-transaminase (Mathew et al. 2017; Bea et al. 2011), nitrilase (Yu et al. 2019), amino acid dehydrogenase (Zhang et al. 2015), ammonia lyase (AL) (Zhang et al. 2020b), lipase (Zeng et al. 2018), and tautomerase (Liu et al. 2020). However, based on the principles of atomic-economy and cost-effectiveness, the most straightforward approach is the use of AL as the catalyst to drive the hydroamination of unsaturated carboxylates (Fibriansah et al. 2011). This mild biocatalytic process would offer a welcome alternative for the synthesis of unnatural amino acids and their derivatives.

ALs, belonging to the enolase family, specifically use unsaturated carboxylates as substrates for the hydroamination reaction to generate unnatural amino 
acids and their derivatives (Turner 2011). Among ALs, MAL has attracted much attention because of its broad substrate specificity and its acceptance of a variety of amines (Leese et al. 2013). Recently, MAL and its variants have been reported to catalyze $\mathrm{C}-\mathrm{N}$ bond formation in the synthesis of artificial dipeptide sweeteners, showing that they possess broad amine scope in accepting unnatural substrates (Zhang et al. 2020b). However, despite these notable advances, unnatural amino acids based on large-frame organic acids remain largely unstudied. They can be envisaged as playing an important role in the synthesis of many chemicals, such as 3,4-dihydroxycinnamic acid, a precursor in the production of levodopa (L-dopa) (Fordjour et al. 2019).

The potential of enzymes to be modified by directed evolution has attracted much attention in recent years (Kramer et al. 2019). Structure-guided protein engineering has replaced the natural mutation of enzymes as one of the main tools of evolution (Cheng et al. 2018; Renata et al. 2015). For example, Wu and coworkers used structure-based computational enzyme design to successfully convert aspartase into a series of complementary hydroamination biocatalysts showing excellent regio- and enantioselectivity (Li et al. 2018). However, the direction of directed evolution is uncontrollable, so the correct selection of the mutagenesis strategies and the amount of screening verification work are key in determining the ultimate degree of success (Zhou et al. 2016). Screening is the bottleneck of directed evolution, and two strategies are presently in use. In the first, the focus is on developing efficient mutagenesis strategies to generate high-quality libraries to minimize the detection workload (Zhou et al. 2016; Hu et al. 2020; Li et al. 2019). In the second, the aim is to establish high-throughput screening methods, allowing rapid discrimination based on colorimetric or optical changes (Li et al. 2019, 2020).

Our previous studies have already led to the mining of an MAL from E. coli O157:H7, which was found to catalyze the hydroamination of short-chain unsaturated acids such as fumaric acid or itaconic acid to the corresponding amino acids with good selectivity. However, a dramatic decrease in reactivity was observed when the substrate side chains increased. Therefore, using structural information as a guide to expand the substrate scope of engineering MALs is the main objective. In this study, we aimed to probe new residues located near the active center and entrance tunnel, which may affect the acceptance of bulky aromatic unsaturated acids. We then further adjusted them to expand the substrate spectrum of MAL.

\section{Materials and methods Chemicals}

PrimerSTAR max DNA polymerase was obtained from TaKaRa Biotechnology Co. (Dalian, China). Escherichia coli strains BL21 (DE3) and DH5 $\alpha$ were bought from TransGen Biotech Co., Ltd. (Beijing, China). All other commercial chemicals were of analytical grade or above, and were purchased from Aladdin (Shanghai, China), Macklin (Shanghai, China), TCI (Tokyo, Japan) or SigmaAldrich (St. Louis, USA).

\section{Homology modeling and molecular docking}

The EcMAL homology model was created using SWISSMODEL (https://swissmodel.expasy.org/), taking MAL from Clostridium tetanomorphum (PDB ID:1KKR) as a template (Lambrughi et al. 2019). The modeled protein structure was evaluated through a Ramachandran plot, and the percentage of residues in the allowed region was 98.6\%. Molecular docking was performed with Yasara using the default program parameters. The center coordinates of the box were determined by visual molecular dynamics, and the box size was set as $20 \AA$ in each dimension. The docking results were selected according to binding affinities and molecular conformations (Cheng et al. 2018). PyMOL was used to display and analyze the modeled protein (Yuan et al. 2016). Amino acids with low conservation score at the entrance of the active pocket were chosen as hotspots (Yu et al. 2019). CAVER 3.0 (http://www.caver.cz) was used to identify the tunnels present in EcMAL (Zhang et al. 2019).

\section{PCR-based methods for site mutagenesis and library construction of ECMAL}

Site-directed mutagenesis and combinatorial active site saturation testing were conducted by the overlap PCR and megaprimer approach with PrimerSTAR max DNA polymerase. The reaction system had a total volume of 50 $\mu \mathrm{L}$, comprising $25 \mu \mathrm{L}$ of PrimerSTAR max polymerase, $0.5 \mu \mathrm{L}(50-100 \mathrm{ng})$ of template DNA, and $200 \mu \mathrm{m}$ primers mix ( $2 \mu \mathrm{L}$ each), made up to the specified volume with water. PCR amplification protocol for short fragments: $98{ }^{\circ} \mathrm{C}$ for $2 \mathrm{~min}$; $\left(98^{\circ} \mathrm{C}\right.$ for $30 \mathrm{~s}, 55^{\circ} \mathrm{C}$ for $30 \mathrm{~s}, 72{ }^{\circ} \mathrm{C}$ for $1 \mathrm{~min}) \times 30$ cycles; $72{ }^{\circ} \mathrm{C}$ for $5 \mathrm{~min}$. PCR products with the above short fragments were then used as primers for mega-PCR: $98^{\circ} \mathrm{C}$ for $2 \mathrm{~min}$; $\left(98^{\circ} \mathrm{C}\right.$ for $30 \mathrm{~s}, 60^{\circ} \mathrm{C}$ for $30 \mathrm{~s}, 72{ }^{\circ} \mathrm{C}$ for $\left.7 \mathrm{~min}\right) \times 30$ cycles; $72{ }^{\circ} \mathrm{C}$ for $10 \mathrm{~min}$. The PCR products were resolved by agarose gel electrophoresis and purified using a Sangon Biotech purification kit. Digestion reaction program: $2 \mu \mathrm{L}$ of NEB CutSmart buffer and $1 \mu \mathrm{L}$ of Dpn I were added for every $20 \mu \mathrm{L}$ of identified PCR product, and the mixture was incubated for $4 \mathrm{~h}$ at $37^{\circ} \mathrm{C}$. After thermal inactivation at $80^{\circ} \mathrm{C}$ for 
20 min, plasmids containing the mutated gene were directly transformed into E. coli BL21 (DE3) and then plated on a Luria-Bertani (LB) agar plate with $100 \mu \mathrm{g} / \mathrm{mL}$ of ampicillin.

Multi-site simultaneous mutagenesis is based on specific amino acid selection and primer design, which determines the size and quality of the constructed library. Here, the 19 residues involved in EcMAL were divided into five groups (A-E). The short fragments between multiple mutation sites, as primers for the next round of amplification reactions, were then amplified by PCR (Wang et al. 2016). The purified short fragments were used as primers to amplify the whole plasmid PET32aEcMAL, leading to the final range of plasmids for library generation. After digestion by Dpn I, the PCR products were transformed into E. coli BL21 (DE3) cells to create a library for screening. Applying standard transformation procedures, the transformants were spread on HyBond- $\mathrm{N}$ membranes, which were then placed on the surface of LB/ampicillin (Amp, $100 \mu \mathrm{g} / \mathrm{mL}$ ) agar plates. After incubation overnight at $37{ }^{\circ} \mathrm{C}$, the membranes were transferred to new $\mathrm{LB} / \mathrm{amp}$ plates with $0.1 \mathrm{mM}$ isopropyl- $\beta$-D-thiogalactopyranoside (IPTG) and incubated for $12 \mathrm{~h}$ at $30{ }^{\circ} \mathrm{C}$. Each membrane was then transferred to a clean plate and stored at $-20^{\circ} \mathrm{C}$ prior to use.

\section{Screening procedures}

Our solid-phase screening procedure is based on the analysis method devised by Turner's group (Aleku et al. 2017). Membranes containing the clones were repeatedly frozen in liquid $\mathrm{N}_{2}$ and thawed four times to lyse the cells. The membranes were then placed on top of a filter paper that was previously loaded with final concentrations of $10 \mathrm{mM}$ screening substrate, $20 \mathrm{mM} \mathrm{Mg}^{2+}$, and $500 \mathrm{mM} \mathrm{NH}_{4} \mathrm{Cl}$ in $500 \mathrm{mM}$ Tris- $\mathrm{HCl}$ buffer $(\mathrm{pH} 8.5)$. After incubation for $6 \mathrm{~h}$ at $30^{\circ} \mathrm{C}$, color-changed colonies were picked and inoculated into LB/amp for subsequent activity detection and mutant sequencing comparison. The number of mutants screened is calculated according to the $95 \%$ mutation coverage determined by mutation toolbox developed by Reezt's team (Reetz et al. 2005).

\section{Protein expression and purification}

Wild-type and positive variants of EcMAL were inoculated in $20 \mathrm{~mL}$ of LB medium containing $100 \mu \mathrm{g} / \mathrm{mL}$ of ampicillin and incubated at $37^{\circ} \mathrm{C}$ and $180 \mathrm{rpm}$. The overnight cultures were transferred into $500 \mathrm{~mL}$ of medium at a $1 \%$ inoculum level. When the absorbance of the medium at $600 \mathrm{~nm}$ (OD600) reached 0.6-0.8, a final concentration of $0.1 \mathrm{mM}$ IPTG was added, and the cultivation temperature was adjusted to $16^{\circ} \mathrm{C}$ to induce protein overexpression. After $20 \mathrm{~h}$, each culture was harvested by centrifuging at $8000 \mathrm{~g}$ for $5 \mathrm{~min}$, and the precipitate was washed twice with $0.9 \% \mathrm{NaCl}$ solution. The cells were resuspended in buffer A (200 mM Tris- $\mathrm{HCl}$ buffer containing $20 \mathrm{mM}$ imidazole, $500 \mathrm{mM} \mathrm{NaCl}, \mathrm{pH} 8.5)$ and disrupted by ultrasonication. The cell lysates were centrifuged at $10,000 \mathrm{~g}$ for $10 \mathrm{~min}$ at $4{ }^{\circ} \mathrm{C}$ to remove insoluble debris. The protein supernatant samples were loaded onto a HisTrap Ni-NTA FF column (GE Healthcare, USA) and eluted with buffer B (in which the imidazole concentration was increased to $250 \mathrm{mM}$ ). The purified proteins were then further desalted by passage through a desalination column (GE Healthcare, USA). The Bradford method was used to determine the protein concentration.

\section{Specific activity and enantioselectivity assays of the wild-type and mutant enzymes}

The kinetic parameters of EcMAL were first measured with varying concentrations of $\mathbf{1 a}(0.25-10 \mathrm{mM})$ and $\mathbf{1 b}$ $(0.2-10 \mathrm{mM})$ as substrates by detecting the initial reaction rate of the protein and curve-fitting according to the Michaelis-Menten equation. The specific activities and stereoselectivities of the EcMAL mutants toward 1a, 1b, and $1 \mathbf{c}$ were then measured using purified enzymes. The reactions were carried out as follows: in a reaction system with a total volume of $2 \mathrm{~mL}$, substrate $1 \mathrm{a}(10 \mathrm{mM})$, $\mathbf{1 b}(10 \mathrm{mM})$, or $\mathbf{1 c}(5 \mathrm{mM})$ in dimethyl sulfoxide $(0.5 \mathrm{~mL})$ and the purified enzyme were mixed with Tris- $\mathrm{HCl}$ (500 mM, pH 8.5). The mixture was shaken at $180 \mathrm{rpm}$ and $30{ }^{\circ} \mathrm{C}$, during which samples were withdrawn at regular intervals and extracted with an equal volume of ethyl acetate. The progress of the reaction was monitored by HPLC analysis and all experiments were conducted in triplicate.

\section{Production of LOPA}

In a mixed system with a reaction volume of $20 \mathrm{~mL}$, the final concentrations of the components were as follows: $10 \mathrm{mM}$ caffeic acid (with 2\% (v/v) DMSO), $20 \mathrm{mM}$ $\mathrm{MgCl}_{2}, 500 \mathrm{mM} \mathrm{NH} \mathrm{Cl}_{4}, 200 \mathrm{mg} / \mathrm{L}$ purified enzyme, and $200 \mathrm{mM}$ Tris- $\mathrm{HCl}$ buffer $(\mathrm{pH}$ 8.5). The reaction flask was shaken at $180 \mathrm{rpm}$ and $30^{\circ} \mathrm{C}$. Samples were regularly withdrawn and analyzed by HPLC.

\section{Results and discussion}

\section{Selection and grouping of key amino acids of Ecmal}

Based on our previous studies, we found that EcMAL could accept a range of short-chain unsaturated carboxylic acids, including fumaric acid, mesaconic acid, and itaconic acid, for the asymmetric synthesis of unnatural amino acids (Ni et al. 2020). However, EcMAL showed low activity in the catalysis of bulky unsaturated carboxylic acids. This difference prompted us to investigate the catalytic mechanism of EcMAL from a structural perspective, with a view to further improve the catalytic 
activity and stereoselectivity toward bulky substrates. We chose caffeic acid (1a) as the model compound for assessing the activity of EcMAL mutants, the basic reaction of which is shown in Additional file 1: Scheme S1. Four aromatic unsaturated carboxylic acids (cinnamic acid (1b), $p$-hydroxycinnamic acid (1c), methylcinnamic acid (1d), and acrylic acid (1e)) with different substituents were also selected. These bulky compounds were selected as substrates because their amination products are important components or building blocks of therapeutic drugs, such as the Parkinson's curative L-dopa. Considering that polyphenols can undergo a rapid color-forming reaction under the action of tyrosinase, we selected $\mathbf{1 a}$ as the screening substrate to assess the efficacies of the enzyme variants. Based on the homologous three-dimensional model of EcMAL, Yasara and CAVER 3.0 were used for molecular docking and substrate tunnel identification, respectively (Liu et al. 2019; Heath et al. 2014). The substrate-binding pocket and substrate tunnel have been demonstrated to have varying degrees of impact on the properties of the enzyme, thereby enhancing the differentiation of directed evolution (De Raffele et al. 2020). We simultaneously considered two groups of residues, those lining the binding pocket $(73,170,172,194,329,384)$ and those surrounding the substrate tunnel $(196,198,199$, 240, 277, 307, 308, 356, 365, 389). Three residues (331, $360,361)$ participating in both regions were also taken into account (Fig. 1).

According to the type and spatial location of the 19 selected residues, we attempted to divide them into five groups, as shown in Fig. 2 and Table 1. Although the use of degenerate NNK codons $(\mathrm{N}=\mathrm{A}, \mathrm{G}, \mathrm{C}, \mathrm{T} ; \mathrm{K}=\mathrm{G})$ for saturation mutation would fully cover all possible amino acids, it is also accompanied by a dramatic increase in the number of screening (Wittmann et al. 2020). The degenerate NDT codons $(\mathrm{D}=\mathrm{A}, \mathrm{G}, \mathrm{T})$ encoding the 12 different types of amino acids (C/F/H/D/N/S/I/G/V/L/Y/R) better meet the requirements of a "smart" library (Reetz et al. 2006). Thus, NDT was considered as a simplified codon for library construction. Because the combination of simultaneous mutations increases exponentially, it is still necessary to screen at least $10^{4}$ levels to achieve the theoretical 95\% coverage (Wang et al. 2017). On this basis, we developed a solid-phase screening method that could rapidly screen $10^{3}$ level transformants through color changes on a single plate. The single colonies that met the requirements were subsequently selected for further activity testing (Tang et al. 2018).

\section{Screening and identification of active Ecmal variants}

First of all, we designed mutation primers according to different mutation groups, and constructed the mutant libraries after amplification (Additional file 1: Figure S1). Using the solid-phase color screening procedure described in the experimental part, we performed highthroughput screening of five mutant libraries (Additional file 1: Figure S2). In the first round of screening, several mutants with activity to substrate 1a were screened from libraries $\mathrm{A}$ to $\mathrm{E}$, and the location and type of mutations were further determined by sequencing. The screening results are listed in Table 2 (M1-M7). Clearly, although the double mutations (M1-M3) showed new trace
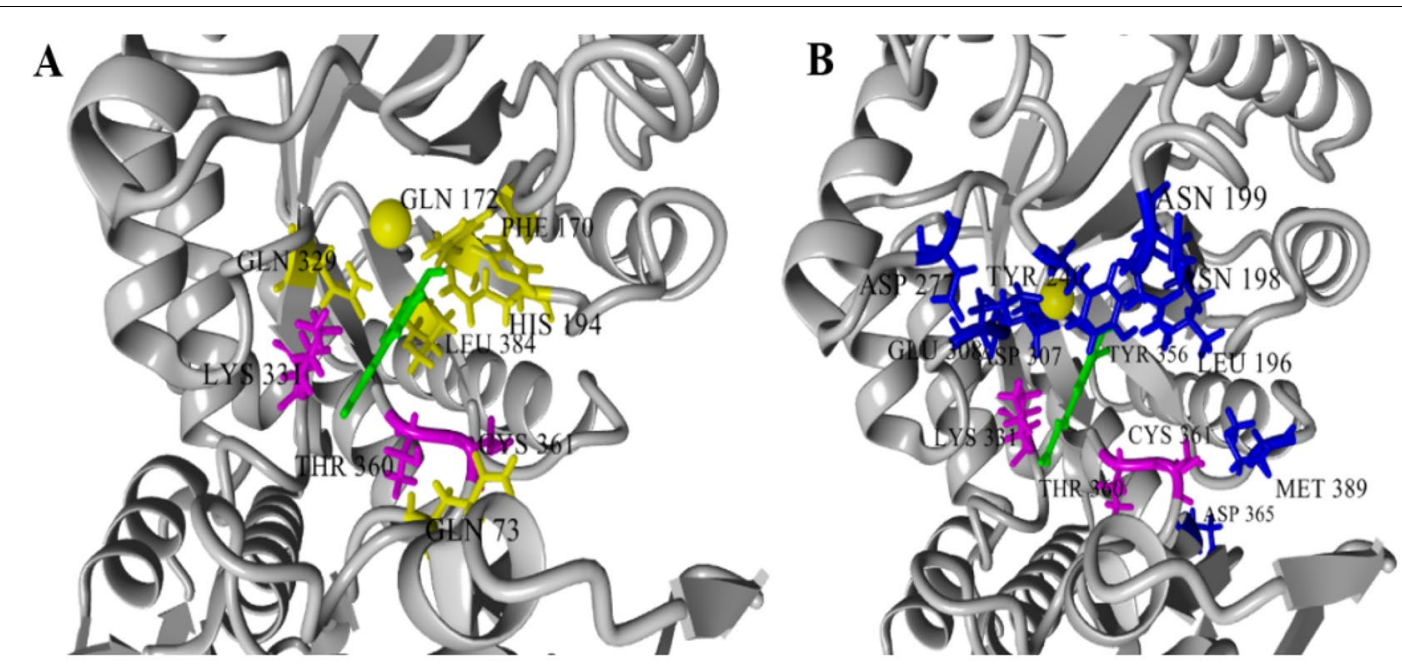

Fig. 1 ECMAL residues chosen for saturation mutagenesis marked in the homology model built by the crystal structure of MAL (PDB: 1 KKR). A Active site mutation sites (yellow) selected on the basis of induced fit docking of amine 1 (green). B Residues surrounding the substrate access tunnel likewise chosen for mutagenesis (blue). The above residues were obtained through the molecular docking of Yasara and the channel recognition function of CAVER 3.0, respectively 

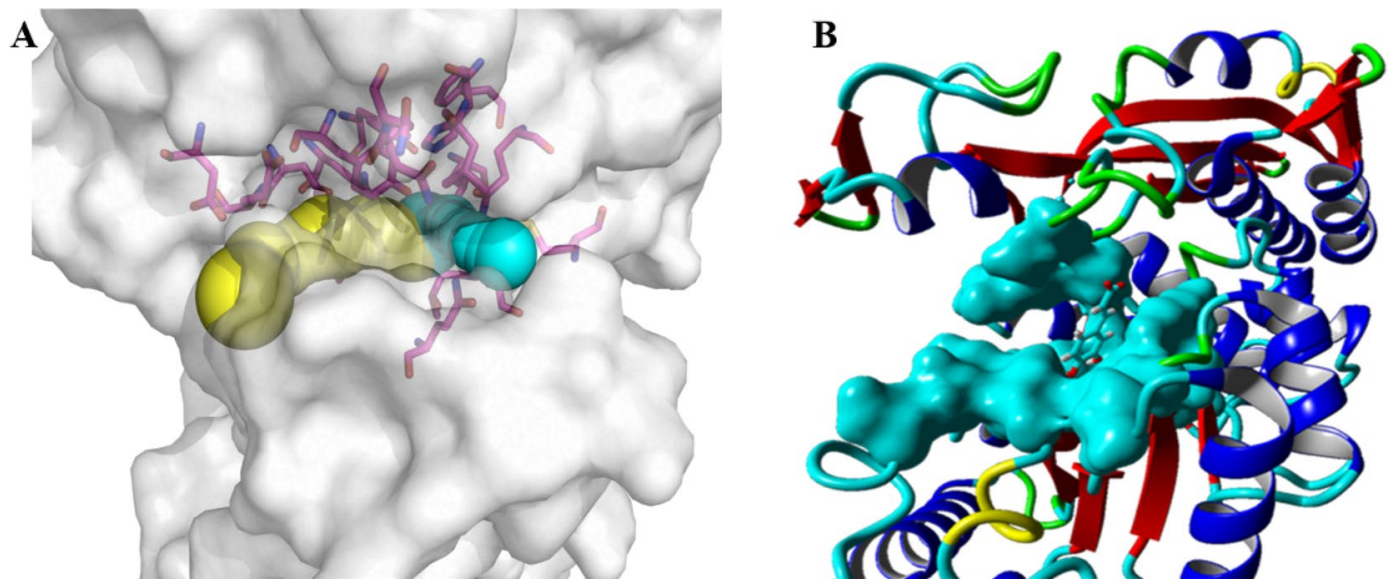

Fig. 2 Spatial structure information of substrate-enzyme docking. A The position relationship between EcMAL channels and the active sites. The target residues of libraries A-E are displayed in purple sticks. The substrate tunnel is shown in yellow and blue. B Poses of caffeic acid docked in the modeled structure of EcMAL. The substrate caffeic acid is shown in azure and the selected residues envelop the substrate in a surface view. The visualization of EcMAL channel, substrate and target amino acid residues was achieved by Pymol and Yasara

Table 1 Grouping of the 19 chosen ECMAL residues into five randomization sites (A, B, C, D and E) and the NDT codes used in saturation mutagenesis

\begin{tabular}{ll}
\hline Randomization site & Code \\
\hline A: $170,172,198,199$ & NDT \\
B: $194,196,384,389$ & NDT \\
C: $307,308,356,360$ & NDT \\
D: 329, 331, 361, 365 & NDT \\
E: 73, 240,277 & NDT \\
\hline
\end{tabular}

The red numbers denote the "active site" positions, the green ones as the "tunnel" positions, and blue ones as the "shared" positions

activity toward caffeic acid compared with the wild type, its activity and stereoselectivity were far from the expectations ( $\mathrm{Li}$ et al. 2019). However, the coincidence that the effective mutants were concentrated in libraries B, $\mathrm{D}$, and $\mathrm{E}$ caught our attention. The triple mutants (M4M6) screened by these libraries further indicated that the selected hot sites in libraries B, D, and E had a great influence on the enzyme to accept bulky substrates. In particular, a quadruple mutation M7 (Q329F/K331R/Y356S/ C361L) suddenly exhibited a mutation transition effect, with remarkable improvements in catalytic activity and selectivity. Motivated by these results, M7 was chosen as the parental enzyme for further iterative combinations. Subsequently, a second round of screening experiments were implemented for libraries B and E using M7 as the template. After screening approximately 5000 clones
Table 2 Properties of the ECMAL variants

\begin{tabular}{llll}
\hline Enzyme and mutants & Mutation sites & $\begin{array}{l}\text { Specific } \\
\text { activity (U/ } \\
\left.\mathbf{g}_{\text {protein }}\right)^{\mathbf{a}}\end{array}$ & \% ee \\
\hline WT ECMAL & None & nd $^{\mathbf{b}}$ & nd $^{c}$ \\
M1 & Q329S/K331R & $5.4 \pm 0.5$ & $16(\mathrm{~S})$ \\
M2 & Q73F/Y240C & $4.4 \pm 0.4$ & $4.4(\mathrm{~S})$ \\
M3 & F170L/E172F & $2.5 \pm 0.5$ & $39(\mathrm{~S})$ \\
M4 & Q329S/K331G/C361C & $6.2 \pm 0.8$ & $86(\mathrm{~S})$ \\
M5 & Q73V/Y240C/D277C & $7.3 \pm 0.1$ & $28(\mathrm{~S})$ \\
M6 & F170S/E172R/D307L & $9.0 \pm 0.3$ & $79(\mathrm{~S})$ \\
M7 & Q329F/K331R/Y356S/ & $26.2 \pm 0.2$ & $99(\mathrm{~S})$ \\
& C361L & & \\
M8 & E172R/Y240S/D307L/ & $38.6 \pm 0.5$ & $99(\mathrm{~S})$ \\
& Q329F/Y356S/C361L & & \\
\hline
\end{tabular}

a Specific activity was determined at $\mathrm{pH} 8.0$ and $30^{\circ} \mathrm{C}$ using purified enzyme and omeprazole sulfide

${ }^{\mathrm{b}}$ The ee values were determined by HPLC

'Not detected

${ }^{\mathrm{d}}$ The bold mutations indicate newly involved mutation(s) in each round

and confirming the specific activities, we identified the best variants (M8) toward the model substrates, which are shown in Table 2. Compared with the catalytic performance identified by other mutants, the M8 presented about 15 -fold improvement and the selectivity was also greatly improved. Considering that M8 was created by partial iterative mutation of $\mathrm{M} 7$, and the catalytic activity was improved while the stereoselectivity was maintained, the results indicate a prominent synergistic influence among these six residues.

To fully investigate the catalytic performance of the selected mutants, several substrates with varying sizes 
and structures, including 1a, 1b, 1c, 1d, and 1e, were selected as candidates for the activity testing of WT and several representative mutants. Notably, there was trace or even no specific activity observed for the WT. Moreover, compared with the bulky caffeic acid, different mutants exhibited obviously different catalytic activities (Ni et al. 2020). Interestingly, the triple site mutant M6 showed significant improvement in activity toward $\mathbf{1 b}$ and was even better than M8 (Fig. 3). The M8 mutant also obviously shows a great advantage in

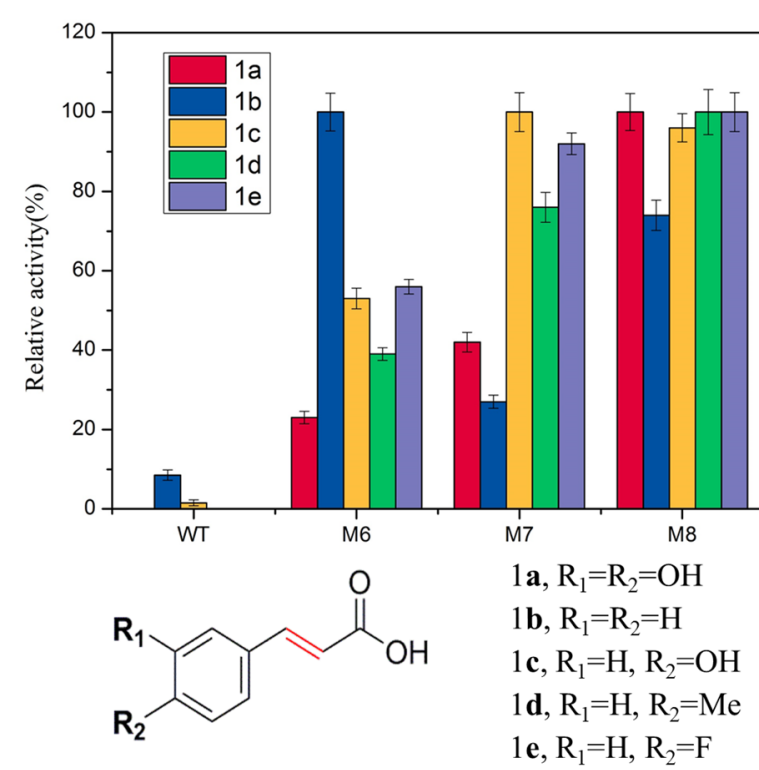

Fig. 3 Activity fingerprints of ECMAL and its variants (M6-M8) with different substrates. The activity was measured by HPLC, and relative activity is measured as a percentage of the optimum activity of each substrate ( $\mathbf{1}$ a caffeic acid, $\mathbf{1} \mathbf{b}$ cinnamic acid, $\mathbf{1} \mathbf{c}$-hydroxycinnamic acid, $\mathbf{1 d}$ methylcinnamic acid, $\mathbf{1 e}$ acrylic acid) accepting other substrates with substituents. These results indicated that the optimal substrate of EcMAL appears at each stage of evolutionary route and the possibility of developing in different catalytic directions.

\section{Structure and computational simulation analysis of EcMAL variants}

To gain insight into the structure-activity relationship of the dramatically altered substrate specificity, the threedimensional structures of $E c \mathrm{MAL}_{\mathrm{WT}}$ and $\mathrm{EcMAL}_{\mathrm{M} 8}$ were first compared by Yasara. As shown in Fig. 4, three polar mutant residues near the active center were replaced with non-polar residues (Q172R, Q329F, C361L) in EcMAL ${ }_{M 8}$, which increased the hydrophobic interaction between the active site and the substrate. Moreover, the residues H194, Q329 and K331 form the catalytic triad (Teze et al. 2020). Among them, the E329 responsible for stabilizing the enolate anion was replaced with phenylalanine, which increased the pi-pi stacked interaction with the substrate. Because the catalytic active center of EcMAL is a wide crack shape, there is theoretically no situation in which the substrate has difficulty in contacting the active center because of the spatial resistance. Therefore, it is speculated that the critical factor affecting the enzyme catalytic activity is the unstable binding between the substrate and enzyme. The docking of the mutant and the substrate also confirmed this view, as the catalytic pocket of the enzyme was more accessible to the substrate.

To investigate the conformational changes of the catalytic active site, whole systems were equilibrated by $\mathrm{MD}$ at $10 \mathrm{~ns}$ without any restriction (Teze et al. 2020). Our goal is to identify conformational changes that regulate the accessibility of the active sites. Based on the snapshots of the MD simulations, it is easy to observe that because of the substitution of phenylalanine at position

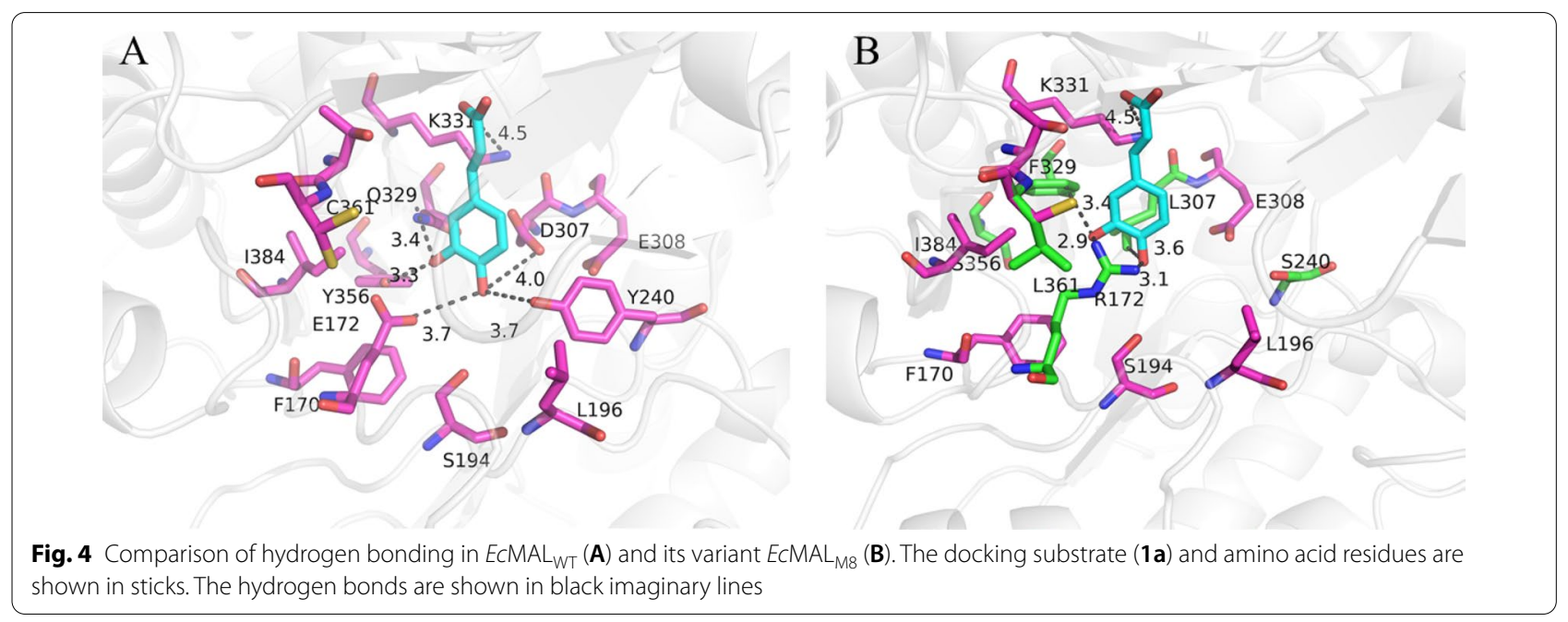


329 , the enhancement in electrostatic attraction to aromatic substrates makes it more accessible to the binding domain. The average per residue root mean square fluctuation (RMSF) of the C $\alpha$ atoms of a monomer in EcMAL was calculated as a measurement of protein flexibility (Additional file 1: Figure S3). Residues L384 and M389 near the tunnel in the $E c M A L_{W T}$ exhibited lower flexibility, which was unfavorable to the bulky substrate in and out of the tunnel. The RMSF changes of the mutant residues also supported this hypothesis.

\section{Catalytic performance and substrate specificity of Ecmal variants}

We subsequently determined the catalytic performance of EcMAL variants for L-dopa synthesis. The engineered EcMALs were used to react under optimal conditions. As shown in Fig. 5, the hydroamination addition reaction catalyzed by variant $\mathrm{M} 8$ reached a conversion rate higher than 50\% within $12 \mathrm{~h}$. Meanwhile, it was also significantly improved compared to other mutants. To further evaluate the specificity responsible for different substrates in EcMAL variants, the kinetic parameters of M6-M8 with substrates 1a-1b were also considered (Table 3). M8 was obtained from M7 through the iterative mutation of two residues 73 and 389. However, it is economical to achieve a large increase in the turnover rate (Kcat from 36.8 to 46.8 ) with a small sacrifice of affinity ( $\mathrm{Km}$ from 1.25 to $1.32 \mathrm{mM}$ ) for substrate 1a. Instead, the best variant for $\mathbf{1 a}$ did not exert higher activity toward $\mathbf{1 b}$, which was equipped with a relatively smaller substituent. M6

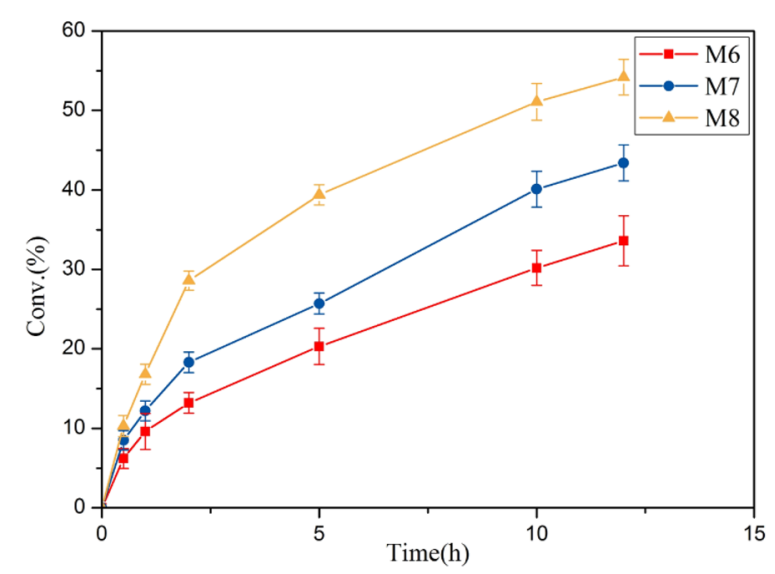

Fig. 5 Progress curves of caffeic acid catalyzed by the purified enzymes of three ECMAL variants (M6, M7, M8). The enzymatic amination addition of caffeic acid $(10 \mathrm{mM})$ was performed at $30^{\circ} \mathrm{C}$ and $\mathrm{pH} 8.5$ with a dose of each $0.5 \mathrm{mg} / \mathrm{mL}$ purified enzymes under the same conditions
Table 3 Kinetic characterization of the ECMAL variants M6-M8 to substrates $\mathbf{1} \mathbf{a}$ and $\mathbf{1} \mathbf{b}$

\begin{tabular}{lllrl}
\hline $\begin{array}{l}\text { Enzyme } \\
\text { and } \\
\text { mutants }\end{array}$ & Substrate & $\boldsymbol{K}_{\mathrm{m}}(\mathbf{m M})$ & $\boldsymbol{K}_{\mathrm{cat}}\left(\mathbf{m i n}^{-\mathbf{1}}\right)$ & $\begin{array}{l}\boldsymbol{K}_{\mathrm{cat}} / \boldsymbol{K}_{\mathbf{m}} \\
\left(\mathbf{m i n}^{-1} \mathbf{m M}^{-1}\right)\end{array}$ \\
\hline M8 & 1a & $1.32 \pm 0.09$ & $61.76 \pm 0.8$ & 46.8 \\
M7 & 1a & $1.25 \pm 0.05$ & $46.02 \pm 0.4$ & 36.8 \\
M6 & 1a & $1.47 \pm 0.05$ & $34.5 \pm 0.74$ & 23.5 \\
M8 & 1b & $1.34 \pm 0.06$ & $31.48 \pm 0.63$ & 23.5 \\
M7 & 1b & $1.96 \pm 0.08$ & $21.5 \pm 0.38$ & 11.0 \\
M6 & 1b & $1.23 \pm 0.03$ & $42.5 \pm 0.72$ & 34.6 \\
\hline
\end{tabular}

${ }^{a}$ Three parallels were measured for each sample. The reaction mixture contained $500 \mathrm{mM}$ Tris- $\mathrm{HCl}$ buffer (pH 8.5), $500 \mathrm{mM} \mathrm{NH}_{4} \mathrm{Cl}, 20 \mathrm{mM} \mathrm{Mg}^{2+}$, 1a: caffeic acid $(0.25-10 \mathrm{mM}) / \mathbf{1}$ b: cinnamic acid $(0.2-10 \mathrm{mM})$

showed strong activity with the lowest $\mathrm{Km}(1.23 \mathrm{mM})$ observed. This may be due to the different epistatic effects of different residues, which simultaneous mutant near the active center and channel of EcMAL, resulting in different non-covalent forces on different substrates.

\section{Conclusion}

In summary, with the aim of activating EcMAL for the amination of bulky unsaturated carboxylic acids, we applied structure-guided strategies for the first time to simultaneously perform saturation mutagenesis on the positions of the residues lining the binding pocket and surrounding the substrate tunnel to successfully evolve the enzyme. Iterative saturation mutagenesis has been applied to optimize the results. Using an effective solid-phase screening method based on the oxidative color-forming reaction of polyphenols by tyrosinase, the effective variants could be quickly screened. Compared with traditional chromatographic screening, this method significantly improves the screening efficiency of the mutant libraries. After several rounds of screening, a series of variants with pronounced increases in catalytic activity and stereoselectivity for bulky substrates (including caffeic acid) were obtained, achieving a substrate specificity that shifted from small to large. Subsequently, the representative mutants were selected to explore the adaptability of bulky substrates by accepting four different substituted aromatic unsaturated carboxylic acids. Meanwhile, the synthesis of levodopa, an anti-dementia drug, was further investigated. The activity of the optimal mutant (M8) to caffeic acid increased by about 15 -fold, and the conversion rate was more than $50 \%$ within $12 \mathrm{~h}$. To gain deeper insight, further study into the crucial residues of engineered enzymes was carried out to explore the regulation mechanism in the substrate spectrum. MD simulation analysis and tunnel prediction were used to 
explore the binding relationship between the substrate and enzyme in bulky substrate catalysis. We look forward to using this work as a starting point to develop more valuable enzymatic hydroamination addition reactions to achieve green and economic synthesis of unnatural amino acids.

\section{Abbreviations}

MAL: Methylaspartate lyase; ECMAL: Methylaspartate lyase from Escherichia coli O157:H7; AL: Ammonia lyase; L-dopa: Levodopa; DMSO: Dimethyl sulfoxide; IPTG: Isopropyl- $\beta$ - $d$-thiogalactoside; MD: Molecular dynamics; RMSF: Root mean square fluctuation.

\section{Supplementary Information}

The online version contains supplementary material available at https://doi. org/10.1186/s40643-021-00456-5.

Additional file 1: Scheme S1. Asymmetric amination of caffeic acid by ECMAL for the production of L-dopa. Figure S1. Primer design and library creation of WT Ec-MAL. Figure S2. Schematic representation of a solidphase screening assay. Figure S3. Molecular dynamics simulation results of ECMAL. Table S1. List of primers for mutation library construction.

\section{Acknowledgements}

We thank Rosalie Tran, Ph.D., from Liwen Bianji (Edanz) (www.liwenbianji.cn/), for editing the English text of a draft of this manuscript.

\section{Authors' contributions}

Conceived and designed the experiments: WYL Performed the experiments: ZFN, PX. Analyzed the data: ZFN. Contributed reagents/materials/analysis tools: MHZ, WYL. Wrote the paper: ZFN, WYL. All authors read and approved the final manuscript.

\section{Funding}

Funding was provided by the National Natural Science Foundation of China (21908070, 21878105), the National Key Research and Development Program of China (2018YFC1603400), the Key Research and Development Program of Guangdong Province (No. 2019B020213001).

\section{Availability of data and materials}

All data generated or analyzed during this study are included in this article.

\section{Declarations}

Ethics approval and consent to participate Not applicable.

\section{Consent for publication}

All authors consent to publishing the manuscript in Bioresources and Bioprocessing. There is no conflict of interest for any of the authors regarding the submission of this manuscript.

\section{Competing interests}

The authors declare that they have no competing interests.

Received: 18 August 2021 Accepted: 8 October 2021

Published online: 19 October 2021

\section{References}

Aleku GA, France SP, Man H, Mangas-Sanchez J, Montgomery SL, Sharma M, Leipold F, Hussain S, Grogan G, Turner NJ (2017) A reductive aminase from Aspergillus oryzae. Nat Chem 9:961-969. https://doi.org/10.1038/ nchem.2782

Bea HS, Park HJ, Lee SH, Yun H (2011) Kinetic resolution of aromatic $\beta$-amino acids by $\omega$-transaminase. Chem Commun 47:5894-5896. https://doi.org/ 10.1039/c1cc11528f

Cheng X, Chen X, Feng J, Wu Q, Zhu D (2018) Structure-guided engineering of: meso-diaminopimelate dehydrogenase for enantioselective reductive amination of sterically bulky 2-keto acids. Catal Sci Technol 8:4994-5002. https://doi.org/10.1039/c8cy01426d

De Raffele D, Martí S, Moliner V (2020) Understanding the directed evolution of de novo Retro-Aldolases from QM/MM studies. ACS Catal 10:7871-7883. https://doi.org/10.1021/acscatal.0c01165

Fibriansah G, Veetil VP, Poelarends GJ, Thunnissen AMWH (2011) Structural basis for the catalytic mechanism of aspartate ammonia lyase. Biochemistry 50:6053-6062. https://doi.org/10.1021/bi200497y

Fordjour E, Adipah FK, Zhou S, Du G, Zhou J (2019) Metabolic engineering of Escherichia coli BL21 (DE3) for de novo production of L-DOPA from D-glucose. Microb Cell Fact. https://doi.org/10.1186/s12934-019-1122-0

Heath RS, Pontini M, Bechi B, Turner NJ (2014) Development of an R-selective amine oxidase with broad substrate specificity and high enantioselectivity. ChemCatChem 6:996-1002. https://doi.org/10.1002/cctc.201301008

Hu Y, Xu W, Hui C, Xu J, Huang M, Lin X, Wu Q (2020) The mutagenesis of a single site for enhancing or reversing the enantio- or regiopreference of cyclohexanone monooxygenases. Chem Commun 56:9356-9359. https://doi.org/10.1039/d0cc03721d

Kramer L, Le X, Hankore ED, Wilson MA, Guo J, Niu W (2019) Engineering and characterization of hybrid carboxylic acid reductases. J Biotechnol 304:52-56. https://doi.org/10.1016/j.jbiotec.2019.08.008

Lambrughi M, Maršić ŽS, Saez-Jimenez V, Mapelli V, Olsson L, Papaleo E (2019) Conformational gating in ammonia lyases. Biorxiv. https://doi.org/10. $1101 / 583088$

Leese C, Fotheringham I, Escalettes F, Speight R, Grogan G (2013) Cloning, expression, characterisation and mutational analysis of L-aspartate oxidase from Pseudomonas putida. J Mol Catal B Enzym 85-86:17-22. https://doi.org/10.1016/j.molcatb.2012.07.008

Li A, Qu G, Sun Z, Reetz MT (2019) Statistical analysis of the benefits of focused saturation mutagenesis in directed evolution based on reduced amino acid alphabets. ACS Catal 9:7769-7778. https://doi.org/10.1021/acscatal. 9 b02548

Li R, Wijma HJ, Song L, Cui Y, Otzen M, Tian Y, Du J, Li T, Niu D, Chen Y, Feng J, Han J, Chen H, Tao Y, Janssen DB, Wu B (2018) Computational redesign of enzymes for regio- and enantioselective hydroamination article. Nat Chem Biol. https://doi.org/10.1038/s41589-018-0053-0

Liu B, Qu G, Li JK, Fan W, Ma JA, Xu Y, Nie Y, Sun Z (2019) Conformational dynamics-guided loop engineering of an alcohol dehydrogenase: capture, turnover and enantioselective transformation of difficult-to-reduce ketones. Adv Synth Catal 361:3182-3190. https://doi.org/10.1002/adsc. 201900249

Liu Y, Xu G, Zhou J, Ni J, Zhang L, Hou X, Yin D, Rao Y, Zhao Y-L, Ni Y (2020) Structure-guided engineering of D-carbamoylase reveals a key loop at substrate entrance tunnel. ACS Catal 10:12393-12402. https://doi.org/10. 1021/acscatal.0c02942

Mathew S, Nadarajan SP, Sundaramoorthy U, Jeon H, Chung T, Yun H (2017) Biotransformation of $\beta$-keto nitriles to chiral (S)- $\beta$-amino acids using nitrilase and $\omega$-transaminase. Biotechnol Lett 39:535-543. https://doi.org/ 10.1007/s10529-016-2271-4

Ni ZF, Zeng YJ, Xu P, Guo ZW, Ou XY, Peng F, Yang JG, Zong MH, Lou WY (2020) Characterization of a novel methylaspartate ammonia lyase from $E$. coli O157:H7 for efficient asymmetric synthesis of unnatural amino acids. ACS Sustain Chem Eng. https://doi.org/10.1021/acssuschemeng.9b05424

Raj H, Szymański W, De Villiers J, Rozeboom HJ, Veetil VP, Reis CR, De Villiers M, Dekker FJ, De Wildeman S, Quax WJ, Thunnissen AMWH, Feringa BL, Janssen DB, Poelarends GJ (2012) Engineering methylaspartate ammonia lyase for the asymmetric synthesis of unnatural amino acids. Nat Chem. https://doi.org/10.1038/nchem.1338

Reetz MT, Bocola M, Carballeira JD, Zha D, Vogel A (2005) Expanding the range of substrate acceptance of enzymes: combinatorial active-site saturation test. Angew Chemie - Int Ed 44:4192-4196. https://doi.org/10.1002/anie. 200500767 
Reetz MT, Wang LW, Bocola M (2006) Directed evolution of enantioselective enzymes: iterative cycles of CASTing for probing protein-sequence space. Angew Chemie Int Ed. https://doi.org/10.1002/anie.200502746

Renata H, Wang ZJ, Arnold FH (2015) Expanding the enzyme universe: accessing non-natural reactions by mechanism-guided directed evolution. Angew Chemie Int Ed 54:3351-3367. https://doi.org/10.1002/anie.20140 9470

Tang CD, Shi HL, Xu JH, Jiao ZJ, Liu F, Ding PJ, Shi HF, Yao LG, Kan YC (2018) Biosynthesis of phenylglyoxylic acid by LhDMDH, a novel D-mandelate dehydrogenase with high catalytic activity. J Agric Food Chem 66:2805-2811. https://doi.org/10.1021/acs.jafc.7b05835

Teze D, Coines J, Raich L, Kalichuk V, Solleux C, Tellier C, André-Miral C, Svensson B, Rovira C (2020) A single point mutation converts GH84 O-GlcNAC hydrolases into phosphorylases: experimental and theoretical evidence. J Am Chem Soc 142:2120-2124. https://doi.org/10.1021/jacs.9b09655

Turner NJ (2011) Ammonia lyases and aminomutases as biocatalysts for the synthesis of $a$-amino and $\beta$-amino acids. Curr Opin Chem Biol 15:234-240. https://doi.org/10.1016/j.cbpa.2010.11.009

Wang JB, Li G, Reetz MT (2017) Enzymatic site-selectivity enabled by structureguided directed evolution. Chem Commun 53:3916-3928. https://doi. org/10.1039/c7cc00368d

Wang JB, Lonsdale R, Reetz MT (2016) Exploring substrate scope and stereoselectivity of P450 peroxygenase OleTJE in olefin-forming oxidative decarboxylation. Chem Commun 52:8131-8133. https://doi.org/10.1039/ c6cc04345c

Wittmann BJ, Knight AM, Hofstra JL, Reisman SE, Jennifer Kan SB, Arnold FH (2020) Diversity-oriented enzymatic synthesis of cyclopropane building blocks. ACS Catal 10:7112-7116. https://doi.org/10.1021/acscatal.0c01888

Wu S, Snajdrova R, Moore JC, Baldenius K, Bornscheuer UT (2020) Biocatalysis: enzymatic synthesis for industrial applications. Angew Chemie Int Ed. https://doi.org/10.1002/anie.202006648

Yu S, Yao P, Li J, Feng J, Wu Q, Zhu D (2019) Improving the catalytic efficiency and stereoselectivity of a nitrilase from: Synechocystis sp. PCC6803 by semi-rational engineering en route to chiral $\gamma$-amino acids. Catal Sci Technol 9:1504-1510. https://doi.org/10.1039/c8cy02455c
Yuan S, Chan HCS, Filipek S, Vogel H (2016) PyMOL and inkscape bridge the data and the data visualization. Structure. https://doi.org/10.1016/j.str. 2016.11.012

Zeng S, Liu J, Anankanbil S, Chen M, Guo Z, Adams JP, Snajdrova R, Li Z (2018) Amide synthesis via aminolysis of ester or acid with an intracellular lipase. ACS Catal 8:8856-8865. https://doi.org/10.1021/acscatal.8b02713

Zhang D, Chen X, Zhang R, Yao P, Wu Q, Zhu D (2015) Development of $\beta$-amino acid dehydrogenase for the synthesis of $\beta$-amino acids via reductive amination of $\beta$-keto acids. ACS Catal 5:2220-2224. https://doi. org/10.1021/cs5017358

Zhang J, Abidin MZ, Saravanan T, Poelarends GJ (2020a) Recent applications of carbon-nitrogen lyases in asymmetric synthesis of noncanonical amino acids and heterocyclic compounds. ChemBioChem 21:2733-2742. https://doi.org/10.1002/cbic.202000214

Zhang J, Grandi E, Fu H, Saravanan T, Bothof L, Tepper PG, Thunnissen AMWH, Poelarends GJ (2020b) Engineered C-N lyase: enantioselective synthesis of chiral synthons for artificial dipeptide sweeteners. Angew Chemie Int Ed 59:429-435. https://doi.org/10.1002/anie.201910704

Zhang Y, Wu YQ, Xu N, Zhao Q, Yu HL, Xu JH (2019) Engineering of cyclohexanone monooxygenase for the enantioselective synthesis of (S)-omeprazole. ACS Sustain Chem Eng 7:7218-7226. https://doi.org/10.1021/acssu schemeng.9b00224

Zhou J, Xu G, Han R, Dong J, Zhang W, Zhang R, Ni Y (2016) Carbonyl groupdependent high-throughput screening and enzymatic characterization of diaromatic ketone reductase. Catal Sci Technol 6:6320-6327. https:// doi.org/10.1039/c6cy00922k

\section{Publisher's Note}

Springer Nature remains neutral with regard to jurisdictional claims in published maps and institutional affiliations.

\section{Submit your manuscript to a SpringerOpen ${ }^{\circ}$ journal and benefit from:}

- Convenient online submission

- Rigorous peer review

- Open access: articles freely available online

- High visibility within the field

- Retaining the copyright to your article

Submit your next manuscript at $\boldsymbol{\nabla}$ springeropen.com 